\title{
Impact of Geriatric Pharmacy Specialist Interventions to Reduce Potentially Inappropriate Medication Among Hospitalized Elderly Patients at Medical Wards: A Prospective Quasi-Experimental Study
}

\author{
Wajanakorn Chivapricha $^{1} \cdot$ Varalak Srinonprasert $^{2} \cdot$ Thanarat Suansanae $^{3}$ (D)
}

Accepted: 26 September 2020 / Published online: 15 October 2020

(c) The Author(s) 2020

\begin{abstract}
Background Elderly patients are at greater risk of receiving potentially inappropriate medications (PIMs) and developing adverse drug events. Identification and correction of PIMs is essential to maximize medication safety.

Objective To determine the prevalence of PIMs on admission in Thai elderly patients admitted to a medical ward and to compare changes of PIMs on discharge, following comprehensive care by a ward pharmacist with or without a geriatric pharmacy specialist.

Patients and method A prospective, quasi-experimental study was performed at a tertiary university hospital in Bangkok, Thailand. Patients aged $\geq 60$ years who were admitted to the medical ward were recruited and allocated to one of two groups: intervention (IG) and control (CG). The CG received pharmaceutical care from the ward pharmacist. The IG received pharmaceutical care from the geriatric pharmacy specialist along with the ward pharmacist. The 2012 Beers criteria were used to identify PIMs on admission, during hospitalization, and on discharge.

Results Prevalence of PIMs on admission was 43.3\% $(N=187)$. On discharge, prevalence of PIMs in the IG decreased significantly compared to that on admission $(21.3 \%$ and $43.3 \%, p<0.05)$ and was significantly lower than in the CG $(21.3 \%$ and $40.9 \%, p=0.036$ ). Moreover, the percentage of patients without PIMs on discharge in the IG was significantly higher than in the CG $(78.7 \%$ and $59.1 \%, p<0.0001)$.

Conclusion Use of PIMs was common among hospitalized elderly patients on admission. Pharmaceutical care provided by a geriatric pharmacy specialist in conjunction with a ward pharmacist significantly reduced the prevalence of PIMs on discharge compared with on admission.
\end{abstract}

\section{Introduction}

Many countries, including Thailand, have a rapidly increasing elderly population. An older population is seven times more likely to be hospitalized due to adverse drug events (ADEs) than a younger population [1,2]. Common risk factors for ADEs in this population include polypharmacy,

Thanarat Suansanae

thanarat.sua@mahidol.ac.th

1 College of Pharmacy, Rangsit University, Pathumthani, Thailand

2 Division of Geriatric Medicine, Department of Medicine, Faculty of Medicine Siriraj Hospital, Mahidol University, Bangkok, Thailand

3 Division of Clinical Pharmacy, Department of Pharmacy, Faculty of Pharmacy, Mahidol University, Bangkok, Thailand inappropriate prescribing, and pharmacokinetic and pharmacodynamic changes $[3,4]$. The majority of adverse drug reactions (ADRs) in older people are 'type A reactions,' which are otherwise preventable. In a prospective study of 1,756 admitted patients aged over 65 years, $45.1 \%$ of ADRs were classified as definitely avoidable and $31.4 \%$ as potentially avoidable [1]. There are several strategies to further reduce this ADE rate, one of which is the identification of potentially inappropriate medications (PIMs).

PIM is defined as a medication for which the potential risk for ADE is higher than the supposed clinical benefit. Dispensing PIMs in the elderly also constitutes suboptimal prescribing, especially if a safer alternative is available. Therefore, several prescribing criteria have been developed to minimize prescribing errors in elderly patients [5-9]. The most referred prescribing criteria, Beers criteria, were developed by the American Geriatric Association based on the expert opinions of geriatric pharmacotherapy specialists 
[10]. In elderly patients, up to $16 \%$ of medications being taken at the time of admission are defined as PIMs, and almost 50\% of admitted patients have at least one PIM [1]. Thus, the identification and management of PIMs might be the best way to prevent ADE in the elderly.

Several studies, focused on the prevalence of PIMs using Beers criteria, have shown that around $42.6 \%$ of prescribed medications were PIMs [11-16]. However, most of these studies were conducted in outpatient settings. The few studies with inpatient settings have demonstrated a different rate of PIMs (around 50-70\%) [12]. Furthermore, a recent meta-analysis found that multi-disciplinary teams involving pharmacists can reduce PIMs by reducing prescribing errors in the older population [13, 14]. Some studies focused on the prevalence of PIMs in elderly patients in Thailand [15], but most of them boasted a retrospective, cross-sectional descriptive design in an outpatient setting; very few studies were carried out at the inpatient unit in primary- or secondary-care hospitals. In addition, these studies used different explicit criteria including Beers criteria and newly developed criteria [16-18].

Therefore, the objective of this study was to determine the prevalence of PIMs in hospitalized elderly Thai patients. In addition, this study aimed to compare the reduction in PIMs on discharge between patients receiving pharmaceutical care delivered by a geriatric pharmacy specialist and patients receiving the usual care by a ward pharmacist.

\section{Methods}

\subsection{Study Design, Setting, and Patient Population}

This was a prospective, quasi-experimental, single-center study conducted at Siriraj Hospital, Bangkok, Thailand from May 2015 to February 2016. All patients aged 60 years or older who were admitted to these medical wards and provided informed consent were included in the study. Patients were excluded if they were admitted for less than $48 \mathrm{~h}$, transferred to other wards, died during admission, or needed either palliative or end-of-life care, as determined by the physician. The study protocol was approved by the review committee of Faculty of Medicine Siriraj Hospital, Mahidol University. Concerning the characteristic of medical wards in our setting, they consisted of eight medical wards with 20 beds in each ward. These wards had been divided into an intervention group (four wards) and a control group (four wards), therefore we expected that there would be 80 patients in each group at the same time. Regarding the criteria for admission, patients were admitted to a vacant bed in each ward assigned by the routine hospital admission protocol; the investigators were not involved in this procedure.
Eligible patients were allocated into either an intervention or a control group depending on their admitted ward. One inpatient ward pharmacist worked regularly with the medical team in each medical ward and one geriatric pharmacy specialist, with a board certified geriatric pharmacotherapy credential, worked among four wards of the intervention group. In the intervention group, the patients were provided pharmaceutical care by the geriatric pharmacy specialist adjunct to the ward pharmacist. In the control group, the patients received standard pharmaceutical care by the ward pharmacist. The standard care included medication reconciliation within $48 \mathrm{~h}$ of patient admission, drug use evaluation, providing drug information to the medical team, and discharge counseling. In addition, the geriatric pharmacy specialist in the intervention group assessed the appropriateness of medication during hospitalization every day, using the 2012 Beers criteria to identify any PIMs and provided a suitable intervention to the physician to optimize drug therapy based on age-related physiological changes and their clinical significance. The team communicated verbally in person or by written notes on the patient chart.

\subsection{Outcome Measurements}

The primary outcome was the prevalence of PIMs on admission. Secondary outcomes included changes in the proportion of PIMs on discharge compared with that on admission between the two groups and changes in each type of PIMs in each group on discharge.

PIMs were identified according to the 2012 Beers criteria by the same geriatric pharmacy specialist throughout the study for both groups. In the intervention group, PIMs were detected via a medical chart review, within $48 \mathrm{~h}$ after admission, every day during hospitalization, and on discharge. In the control group, however, the PIMs were identified retrospectively by a medical chart review after the patients were discharged. The identified PIMs in both groups were classified into three subtypes for subgroup analysis: general PIMs, condition-associated PIMs, and PIMs with anticholinergic properties.

Furthermore, all patients in both groups were followed up via phone call by the geriatric pharmacy specialist at 2 weeks after discharge to assess medication adherence, possible adverse events, and other potential medical and drug-related problems. If drug-related problems such as side effects were identified, the geriatric pharmacy specialist provided solutions to the patients and their caregivers. However, if a serious medical illness was found, rehospitalization or an outpatient visit was recommended. 


\subsection{Sample Size Calculation and Statistical Analysis}

The sample size was calculated based on the results obtained from Egger et al. [19], which revealed that the estimated prevalence of PIMs in hospitalized elderly patients was 0.16 . Thus, at least 207 patients had to be recruited for a significance level of $\alpha=0.05$ and a power of at least $80 \%(\beta<0.20)$.

All data were tested for normal distribution by measuring kurtosis and skewness. The baseline characteristics were compared between groups by using Fisher's exact test for nominal data and the Mann-Whitney $U$ test for continuous data. The primary analytical approach was a per-protocol analysis. Descriptive analysis was applied for the number of PIMs. The $\chi^{2}$ test was used to compare the prevalence of PIMs between groups on admission, during hospitalization, and on discharge. A paired $t$ test was used to compare the prevalence of PIMs on discharge with that on admission in the same group. McNemar's test was used to test the nominal data. A $p$ value of less than 0.05 was considered to indicate a statistically significant difference. All analyses were performed using the Statistical Package for the Social Sciences for Windows version 21.

\section{Results}

\subsection{Baseline Characteristics}

In total, 234 patients were enrolled, of whom 47 patients (20\%) were excluded according to the exclusion criteria. Of these, two were admitted for less than $48 \mathrm{~h}$, three were transferred to other wards, 39 died during admission, and three needed either palliative or end-of-life care, as determined by the physician. Then, the 187 eligible patients were divided into two groups with 94 and 93 patients in the intervention and control groups, respectively. There was no significant difference between the baseline characteristics of the patients in both groups (Table 1). The mean age of the patients in the intervention and control group was $74.0 \pm 8.4$ and $74.9 \pm 9.0$ years, respectively. Both groups had more than $50 \%$ male population and their functional status could be identified as partially dependent. The average number of prior medications per patient on admission was $7.8 \pm 4.4$ and $8.7 \pm 5.1$ items in the intervention and control groups, respectively. The most common causes of hospitalization for both groups were heart failure, pneumonia, non-ST segment elevation myocardial infarction, chronic obstructive pulmonary disease with acute exacerbation, and upper gastrointestinal bleeding.

Table 1 Baseline characteristics of patients in the intervention group and control group $(N=187)$

\begin{tabular}{lll}
\hline Characteristic & Intervention group $(n=94)$ & Control group $(n=93)$ \\
\hline Age (years), mean \pm SD & $74.0 \pm 8.4$ & $74.7 \pm 9.0$ \\
$60-74$ years, $n(\%)$ & $57(60.6)$ & $46(49.5)$ \\
$75-84$ years, $n(\%)$ & $24(25.5)$ & $30(32.3)$ \\
$\geq 85$ years, $n(\%)$ & $13(13.8)$ & $17(18.3)$ \\
Sex: male, $n(\%)$ & $48(51.1)$ & $48(51.6)$ \\
BMI $\left(\mathrm{kg} / \mathrm{m}^{2}\right)$, mean $\pm \mathrm{SD}$ & $21.9 \pm 3.9$ & $22.58 \pm 4.0$ \\
Functional status & & $20(21.5)$ \\
Independent, $n(\%)$ & $29(30.9)$ & $52(55.9)$ \\
Partially dependent, $n(\%)$ & $41(43.6)$ & $21(22.6)$ \\
Totally dependent, $n(\%)$ & $24(25.5)$ & $12(12.9)$ \\
Cause of admission, $n(\%)$ & & $7(7.5)$ \\
Heart failure & $11(11.7)$ & $6(6.5)$ \\
Pneumonia & $15(16)$ & $5(5.4)$ \\
NSTEMI & $6(6.4)$ & $3(3.2)$ \\
COPD with AE & $5(5.3)$ & $8.7 \pm 5.1(9)$ \\
UGIB & $6(6.4)$ & 0.940 \\
Average number of medications prior to admission, & $7.8 \pm 4.4(8)$ & $13.41,10(3-51)$ \\
mean \pm SD (mode) & & 0.207 \\
Length of hospital stay (days), mean, median (range) & $16.97,14(3-78)$ & 0.157 \\
\hline
\end{tabular}

$B M I$ body mass index, $C O P D$ with $A E$ chronic obstructive pulmonary disease with acute exacerbation, $N S T E M I$ non-ST segment elevation myocardial infarction, UGIB upper gastrointestinal bleeding 
Table 2 Prevalence of potentially inappropriate medications (PIMs) during the study

\begin{tabular}{llll}
\hline & Admission (\%) & $\begin{array}{l}\text { Hospi- } \\
\text { talization } \\
(\%)\end{array}$ & Discharge (\%) \\
\hline Total $(N=187)$ & 43.3 & 46.0 & $31.0^{*}$ \\
$\begin{array}{l}\text { Intervention group } \\
(n=94)\end{array}$ & 43.6 & 43.6 & $21.3^{* *}$ \\
Control group $(n=93)$ & 43.0 & 48.4 & 40.9 \\
\hline
\end{tabular}

$* p<0.05$ compared between the prevalence of PIMs on discharge and on admission

$* * p=0.036$ compared between intervention group and control group on discharge

Table 3 Potentially inappropriate medication (PIM) classes on admission $(N=187)$

\begin{tabular}{lc}
\hline Medication/medication class & Percentage \\
\hline Benzodiazepines & 21.70 \\
Doxazosin & 16.04 \\
Orphenadrine + paracetamol & 7.55 \\
Amitriptyline & 6.60 \\
NSAIDs & 5.66 \\
Hydroxyzine & 5.66 \\
Trihexyphenidyl & 4.72 \\
Cyproheptadine & 3.77 \\
Methyldopa & 2.83 \\
Megestrol & 2.83 \\
\hline
\end{tabular}

NSAIDs non-steroidal anti-inflammatory drugs

\subsection{Prevalence of Potentially Inappropriate Medications (PIMs)}

We found that 81 patients took at least one PIM on admission; thus, the overall prevalence of PIMs on admission was 43.3\% (intervention group: $43.6 \%$, control group: $43.0 \%$ ), with no significant difference between the groups $(p=0.636)$ (Table 2). The most common PIM classes are summarized in Table 3.

The prevalence of PIMs during hospitalization slightly increased but had no significant difference when compared with the prevalence of PIMs on admission in both groups.

On discharge, the overall prevalence of PIMs was found to be significantly decreased from that on admission (31.0\% and $43.3 \%$, respectively, $p<0.05$ ). Moreover, the prevalence of PIMs in the intervention group was found to be significantly lower than that in the control group $(21.3 \%$ and $40.9 \%$, respectively, $p=0.036$ ). Consequently, the percentage of patients without PIMs in the intervention group was significantly higher than that in the control group $(78.7 \%$ and $59.1 \%$, respectively, $p<0.0001$; Fig. 1 ).

\subsection{Types of PIMs Through the Hospital Course}

On admission, the most prevalent type of PIMs found in both groups was general PIMs (56.7\% vs. 57.4\% in the intervention and control group, respectively; Fig. 2). The prevalence of condition-associated PIMs and PIMs with anticholinergic properties in the intervention group was found to be $10.0 \%$ and $33.3 \%$, respectively. In contrast, in the control group, the prevalence of PIMs was found to be $14.8 \%$ and $27.9 \%$, respectively. Overall, there was no significant difference in the prevalence of each type of PIM between groups.

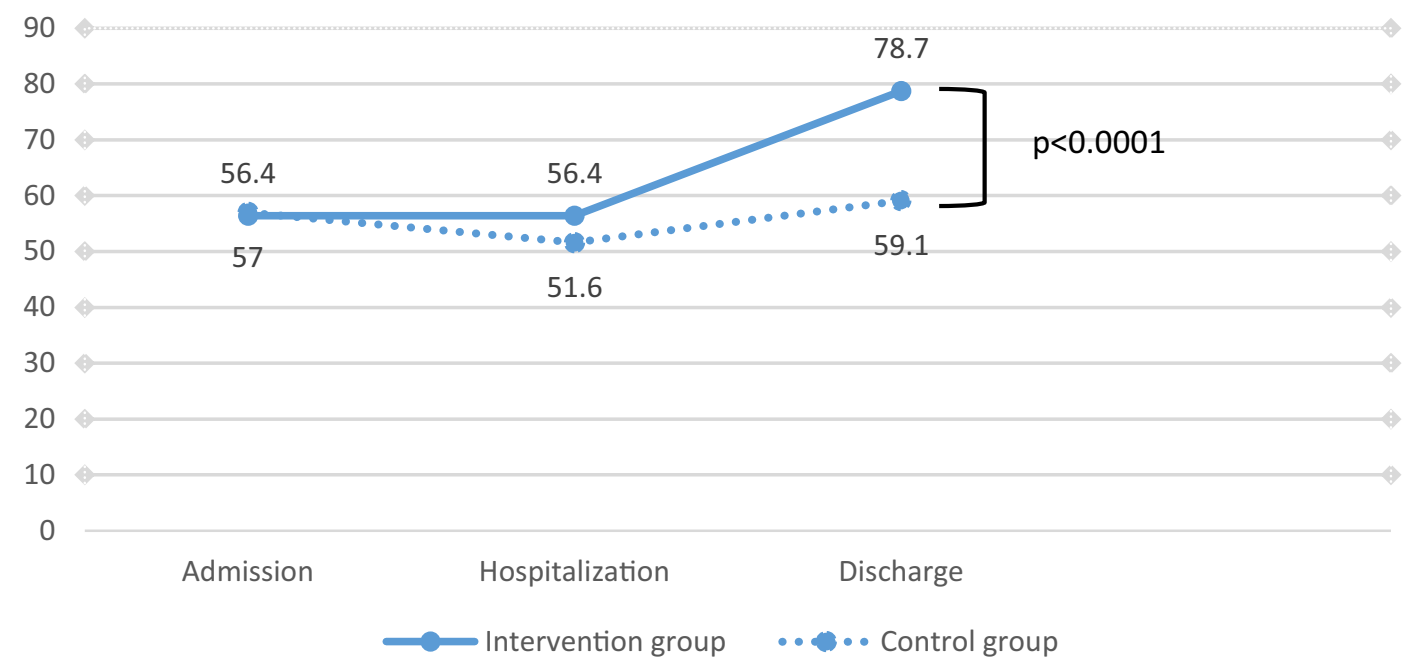

Fig. 1 Percentage of patients without potentially inappropriate medications (PIMs) on admission, during hospitalization and on discharge 


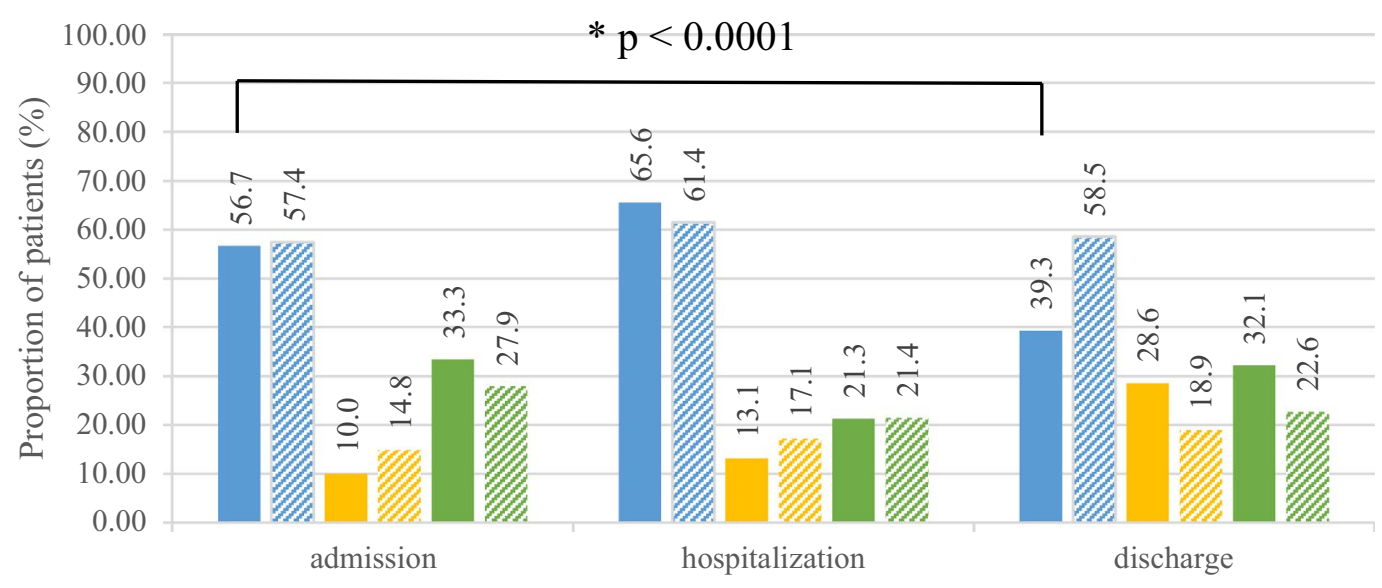

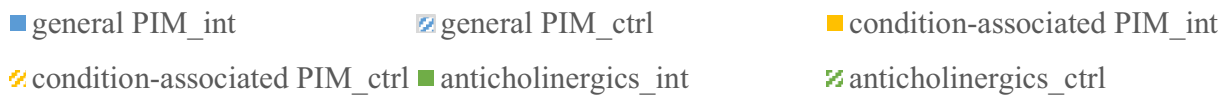

Fig. 2 Prevalence of potentially inappropriate medications (PIMs) in the intervention group and control group on admission, during hospitalization, and on discharge. PIM_int PIMs in intervention group,

During hospitalization, the prevalence of general PIMs and condition-associated PIMs slightly increased in both groups (Fig. 2). In contrast, the prevalence of PIMs with anticholinergic properties declined in both groups. However, the prevalence of each type of PIM showed no statistical difference when compared with that on admission.

On discharge, the prevalence of general PIMs in the intervention group was found to be significantly decreased when compared with that on admission $(39.3 \%$ vs. $56.7 \%$, respectively, $p<0.0001)$. In contrast, this prevalence in the control group showed no difference from that on admission (58.5\% vs. $57.4 \%$, respectively). The prevalence of condition-associated PIMs increased during hospitalization in both groups but did not show any statistically significant difference when compared with that on admission. The prevalence of PIMs with anticholinergic properties did not show much difference in the intervention group from that on admission, while it decreased in the control group from that on admission, but without a significant difference.

All patients found to be prescribed PIMs on discharge in the intervention group $(n=20)$ and the control group $(n=38)$ were scheduled to have a health assessment by phone call 2 weeks after being discharged from the hospital. The results showed that some patients in both groups experienced adverse events, such as dizziness, sedation, and headache, with no need for medication management (Table 4). Only three patients in the intervention group and six patients in the control group developed serious adverse events, which needed medical attention. One patient in the
PIM_ctrl PIMs in control group. *Significant difference between on admission and discharge $(p<0.05)$

Table 4 Clinical outcomes at 2 weeks after discharge from hospital in patients who had potentially inappropriate medications (PIMs) on discharge

\begin{tabular}{lll}
\hline Number of patients & $\begin{array}{l}\text { Interven- } \\
\text { tion group } \\
(n=94)\end{array}$ & Control group $(n=93)$ \\
\hline $\begin{array}{l}\text { Patients with no PIMs on } \\
\text { discharge }\end{array}$ & 74 & 55 \\
$\begin{array}{l}\text { Patient had at least one PIM } \\
\text { on discharge }\end{array}$ & 20 & 38 \\
$\begin{array}{l}\text { Average number of PIMs on } \\
\text { discharge (mean } \pm \text { SD) }\end{array}$ & $1.25 \pm 0.77$ & $1.32 \pm 0.62$ \\
$\begin{array}{l}\text { No adverse events } \\
\text { Developed adverse events } \\
\text { but no need for other } \\
\text { treatment }\end{array}$ & $7(35.0 \%)$ & $14(36.8 \%)$ \\
$\begin{array}{c}\text { Developed adverse events } \\
\text { and need medication } \\
\text { management }\end{array}$ & $3(15.0 \%)$ & $6(15.8 \%)$ \\
Rehospitalization & $1(5.0 \%)$ & $2(5.3 \%)$ \\
\hline
\end{tabular}

intervention group developed delirium from anticholinergic usage (cyproheptadine) and one patient in the control group experienced confusion and disorientation, due to a suspected acute fever, requiring rehospitalization. Another patient in the control group with a history of myocardial infarction developed acute heart failure after taking 
non-steroidal anti-inflammatory drugs (NSAIDs) obtained from a drug store and required rehospitalization.

\section{Discussion}

All patients enrolled in the study were allocated to the wards by the inpatient unit officer, depending on their physician and availability of units in each ward. Therefore, this study had a quasi-experimental design, as true randomization was not possible. In addition, each medical ward at the study site was operated separately. We ensured that the ward pharmacist provided identical pharmaceutical care in each ward every day that complied with the standard care of pharmacy professionals. However, the results from our unpublished preliminary observational study showed that PIMs were still detected in the prescription of elderly patients who were discharged from these wards. Consequently, a geriatric pharmacy specialist with expert knowledge and experience in geriatric pharmacotherapy could help to fill the gap in standard care delivered by the ward pharmacist in the medical ward [20]. Therefore, this may be the first study to verify the impact of geriatric pharmacy specialist involvement on the prevalence of PIMs in hospitalized elderly patients.

In our study, we used 2012 Beers criteria to identify PIMs. Thai healthcare professionals, including physicians and pharmacists, are more familiar with the Beers criteria than others. Thus, lack of knowledge about medication lists to be avoided in the elderly could not be given as a reason. Furthermore, the ward pharmacist informed the relevant physician about the appropriateness of medication by referring to the Beers criteria for elderly patients. Accordingly, we could assess the impact of the geriatric pharmacy specialist on the administration of PIMs during hospitalization when compared with the impact of ward pharmacist in the control group. This may also explain the reduction of PIMs in the control group during hospitalization and on discharge, especially PIMs with anticholinergic properties.

Data from 187 eligible patients were analyzed, and this number was lower than the expected sample size $(N=207)$. This figure was not used to determine the impact of interventions between intervention and control groups. The results of our study demonstrated that PIMs could be detected during the study period in both groups. Therefore, the reduced number of participants should not have affected to the overall results.

Regarding PIMs detection, we found that $43.3 \%$ of our patients were taking at least one PIM on admission. This finding is comparable with that of previous studies, where the prevalence of PIMs in hospitalized patients ranged between $44.4 \%$ and $79.0 \%$ [21, 22], and this range of values is higher than that in the outpatient setting (16.0-53.0\%) [6, 23-25]. Our study could not be considered a pioneer study in terms of studying the prevalence of PIMs in Thailand. However, previous studies that have assessed PIMs in elderly Thai patients have been mostly performed in an outpatient setting $[15,26]$. The reported prevalence of PIMs was in the range of $19.2 \%$ and $28.1 \%$, which is relatively smaller than that in our study [15]. Moreover, the PIMs on admission in our study were calculated from the lists of medications prior to admission, and should not have differed from those in outpatient settings. However, the comparison with other studies must be interpreted carefully to account for differences in the patient settings, criteria used to detect PIMs, and the study methodology employed.

The most commonly observed PIMs in our study were benzodiazepines, such as diazepam, lorazepam, clonazepam, and NSAIDs, comparable to other studies [6, 27]. However, our results also showed doxazosin, an alpha-1 receptor antagonist, as a common PIM, which diverged from previous study results. Doxazosin is the treatment of choice for symptomatic benign prostatic hypertrophy, the most common prostate problem for older men [28]. As half of our eligible participants were male (51\%) and over 60 years old, they were likely to receive doxazosin for this indication. However, doxazosin has a high risk of orthostatic hypotension and is not recommended as a routine treatment for patients with hypertension [29]. In addition, a safer alpha-1 receptor antagonist with highly selective and subtype-specific, such as tamsulosin, is available, which is generally better tolerated than other alpha-1 receptor antagonists (terazosin, prazosin, doxazosin) and has no clinically relevant effect on blood pressure [29, 30].

With regards to the prevalence of PIMs on admission between groups, no statistically significant difference in the prevalence of PIMs, and the number of general PIMs, condition-associated PIMs, and PIMs with anticholinergic properties was observed between the study groups. This might be related to the similarity in the baseline characteristics between the intervention and control groups, particularly the number of medications on admission.

During hospitalization, the prevalence of total PIMs in both groups counted cumulatively across the hospital stay did not show a significant difference when compared with that on admission. However, there was a relatively higher prevalence of general PIMs and condition-associated PIMs in both groups than that on admission. Notably, the oral long-acting benzodiazepines, NSAIDs, and medication with anticholinergic properties, which were commonly identified PIMs on admission, had been discontinued on the recommendation of the ward pharmacist after completion of medication review and reconciliation. In addition, the geriatric pharmacy specialist recommended discontinuation 
of long-acting sulfonylurea (glyburide) and central alpha-1 receptor agonist (methyldopa) in the intervention group. The ward pharmacist and the physician may have been unaware of these medications being PIMs. Nevertheless, some new general PIMs were documented during hospitalization, especially at night time, when neither the ward pharmacist nor geriatric pharmacy specialist worked at the ward. Examples of these PIMs were meperidine injection for severe acute pain, oral lorazepam for insomnia and anxiety, oral metoclopramide for nausea, and intravenous diazepam for status epilepticus. Noticeably, most of these medications have safer alternatives except for status epilepticus [31, 32]. Similarly, using oral short- to intermediate-acting benzodiazepine for short-term symptomatic treatment of acute anxiety (not more than 2 weeks) was considered appropriate [33]. We believe that these recognized PIMs were continued during hospitalization and at discharge because the physician considered that their benefit outweighed their short-term risk.

Condition-associated PIMs was determined according to a list of medications that should be avoided in older adults with certain diseases. The increased number of conditionassociated PIMs in the intervention group was related to antipsychotics. Antipsychotics should be avoided in patients with dementia or cognitive impairment because they can increase the risk of cerebrovascular events. In our study, dementia was found in $4.3 \%$ and $3.3 \%$ of the patients in the intervention and control groups, respectively. Thus, antipsychotics, such as risperidone, quetiapine, and olanzapine, were used to treat behavioral and psychological symptoms of dementia such as agitation and delusion which usually flared up with stress, change in daily routines, excessive stimulation and change in environment. Accordingly, these antipsychotic agents were prescribed during hospitalization and extended until discharge.

In contrast, the PIMs with anticholinergic properties during hospitalization were satisfactorily decreased in both groups when compared with on admission. This may be a result of the healthcare professionals' familiarity with the Beers criteria, which recommend avoiding anticholinergic drugs in elderly patients. Therefore, medications with anticholinergic properties found during the medication review (such as hydroxyzine, orphenadrine, and trihexyphenidyl) were recognized by the physician and the ward pharmacist and generally discontinued if there was no specific therapeutic indication. On the other hand, PIMs with anticholinergic properties on discharge were associated with the increasing use of loratadine, which was a non-sedating antihistamine for treatment of allergic symptoms. Loratadine was classified as a PIM with anticholinergic properties according to the 2012 Beers criteria. Nevertheless, it is removed from the list in updated 2015 Beers criteria.

Similarly, on discharge, the prevalence of PIMs in the intervention group was significantly lower than that on admission, whereas only a tiny reduction of total PIMs in the control group was observed when compared with that on admission. Correspondingly, a statistically significant difference in the percentage of patients without PIMs on discharge was observed between the two groups. To our knowledge, PIMs are associated with an increased risk of poor outcomes in the older population including adverse events, drug-drug or drug-disease interaction, and hospitalization $[34,35]$. Accordingly, the patients in the intervention group had better outcomes compared with those in the control group, owing to medication safety. Regardless, there were no established data to demonstrate the relationship between the presence of PIMs on discharge and the risk of adverse effects or rehospitalization rate [36-39]. In our study, the telephone follow-up at 2 weeks post-discharge by the geriatric pharmacy specialist revealed that most of the patients were healthy, despite having PIMs on discharge, while some patients developed adverse events despite having no PIMs on discharge. This finding indicated that, in addition to identifying PIMs to assess medication safety, the management of individualized pharmacokinetic and pharmacodynamic changes, renal function, hepatic function, co-morbidities, and concurrent medications providing recommendations for dose adjustment and discharge counseling plan should also be included in the care protocol to maximize the safety of medication use.

Regarding the prevalence of PIMs subtype on discharge in the intervention group, there was a 31\% reduction of general PIMs compared with that on admission. Although there was an observed increase in general PIMs during hospitalization as discussed above, these new PIMs were prescribed as a once-a-day regimen. Thus, these identified PIMs were withdrawn on discharge. In addition, other medications accounting for general PIMs had been discontinued since admission. Therefore, the prevalence of general PIMs on discharge was found to be dramatically decreased. In contrast, no significant changes in the prevalence of condition-associated PIMs and PIMs with anticholinergic properties on discharge were observed compared with on admission, despite intervention by the geriatric pharmacy specialist. This non-acceptance of pharmacist interventions might be a result of the inpatient healthcare physician's incomplete understanding of the patient's previous illness and medication history. Furthermore, some PIMs were prescribed by specialists or senior physicians, which inpatient physicians are often reluctant to modify or stop even pharmacist's recommendation.

In summary, our study results disclosed that including a geriatric pharmacy specialist in the medical team had an impact on the prevalence of PIMs in hospitalized elderly patients, specifically general PIMs. In addition, our study also emphasized the importance of having a ward pharmacist in the medical team, as they help in complete medication review and reconciliation, including modification or discontinuation of PIMs in the hospitalized elderly patients, especially PIMs with anticholinergic properties. Nevertheless, 
other healthcare professionals including physicians and nurses may help to diminish PIMs as well, provided that they are trained on rational drug use in the elderly. The presence of a pharmacist as a drug expert in the medical team will, however, help reinforce the optimization of drug therapies for elderly patients. Additionally, providing the ward pharmacist with a short training course related to geriatric pharmacotherapy might improve their competency and reduce the prevalence of PIMs, resembling the impact made by a geriatric pharmacy specialist.

There are some obvious limitations to our study. First, several enrolled patients were excluded from the study, and this number was more than that anticipated. This may be related to our study setting being the biggest university hospital in Bangkok. Thus, majority of the patients admitted to our hospital, with co-morbidities and moderate-to-severe disease severity, had to be excluded. Second, we could not assess whether the adverse events that occurred after discharge were correlated with the residual PIMs at discharge. Third, the PIMs identification in the control group was carried out retrospectively after the patient was discharged. Consequently, medication administration records or data related to drug therapy were unavailable or incomplete, thus, such PIMs could not be counted and the prevalence of PIMs could be underestimated.

\section{Conclusion}

The Beers-related PIMs use was highly prevalent among Thai patients aged over 60 years who were hospitalized in the medical ward. Applied geriatric pharmacotherapy knowledge and pharmaceutical skills delivered by a geriatric pharmacy specialist together with the ward pharmacist has the potential to reduce the prevalence of PIMs in this population, as demonstrated by significantly less PIMs on discharge than that on admission. The general PIMs and PIMs with anticholinergic properties are the most common subtypes that can be optimized. However, a shortage of ward pharmacists, especially geriatric pharmacy specialists, may pose a big challenge for the implementation of this model in the general inpatient setting.

Acknowledgements The authors would like to express their great appreciation to all participants, physicians, nurses, ward pharmacists, and all medical staffs at the study wards and those who helped them to complete this study.

\section{Declarations}

Funding Not applicable.

Conflict of interest The authors declare that there are no conflicts of interest.
Ethics approval The study protocol was approved by the review committee of Faculty of Medicine Siriraj Hospital, Mahidol University.

Consent to participate Signed consent forms were obtained from all eligible patients.

Consent for publication Not applicable.

Availability of data and material A subsection on data described in the manuscript, including all relevant raw data, will be freely available to any researcher wishing to use them for non-commercial purposes, without breaching participant confidentiality.

Code availability Not applicable.

Author contributions Conceptualization: WC, VS, TS; methodology: WC, VS, TS; formal analysis and investigation: WC, VS, TS; writingoriginal draft preparation: WC, TS; writing — review and editing: TS; resources: VS, TS; supervision: VS, TS.

Open Access This article is licensed under a Creative Commons Attribution-NonCommercial 4.0 International License, which permits any non-commercial use, sharing, adaptation, distribution and reproduction in any medium or format, as long as you give appropriate credit to the original author(s) and the source, provide a link to the Creative Commons licence, and indicate if changes were made. The images or other third party material in this article are included in the article's Creative Commons licence, unless indicated otherwise in a credit line to the material. If material is not included in the article's Creative Commons licence and your intended use is not permitted by statutory regulation or exceeds the permitted use, you will need to obtain permission directly from the copyright holder. To view a copy of this licence, visit http://creativecommons.org/licenses/by-nc/4.0/.

\section{References}

1. Franceschi M, Scarcelli C, Niro V, Seripa D, Pazienza AM, Pepe G, Colusso AM, Pacilli L, Pilotto A. Prevalence, clinical features and avoidability of adverse drug reactions as cause of admission to a geriatric unit: a prospective study of 1756 patients. Drug Saf. 2008;31:545-56.

2. Davies EA, O'Mahony MS. Adverse drug reactions in special populations - the elderly. Br J Clin Pharmacol. 2015;80:796-807.

3. Mangoni AA, Jackson SHD. Age-related changes in pharmacokinetics and pharmacodynamics: basic principles and practical applications. Br J Clin Pharmacol. 2004;57:6-14.

4. Onder G, van der Cammen TJ, Petrovic M, Somers A, Rajkumar C. Strategies to reduce the risk of iatrogenic illness in complex older adults. Age Ageing. 2013;42:284-91.

5. O'Mahony D, O'Sullivan D, Byrne S, O'Connor MN, Ryan C, Gallagher P. STOPP/START criteria for potentially inappropriate prescribing in older people: version 2. Age Ageing. 2015;44:213-8.

6. Lozano-Montoya I, Vélez-Diaz-Pallarés M, Delgado-Silveira E, Montero-Errasquin B, Cruz Jentoft AJ. Potentially inappropriate prescribing detected by STOPP-START criteria: are they really inappropriate? Age Ageing. 2015;44:861-6.

7. Hanlon JT, Schmader KE, Samsa GP, Weinberger M, Uttech KM, Lewis IK, Cohen HJ, Feussner JR. A method for assessing drug therapy appropriateness. J Clin Epidemiol. 1992;45:1045-51. 
8. Hanlon JT, Schmader KE. The medication appropriateness index at 20: where it started, where it has been, and where it may be going. Drugs Aging. 2013;30:893-900.

9. O'Connor MN, Gallagher P, O'Mahony D. Inappropriate prescribing: criteria, detection and prevention. Drugs Aging. 2012;29:437-52.

10. Campanelli CM. American Geriatrics Society updated Beers criteria for potentially inappropriate medication use in older adults: the American Geriatrics Society 2012 Beers Criteria Update Expert Panel. J Am Geriatr Soc. 2012;60:616-31.

11. Davidoff AJ, Miller GE, Sarpong EM, Yang E, Brandt N, Fick DM. Prevalence of potentially inappropriate medication use in older adults using the 2012 Beers criteria. J Am Geriatr Soc. 2015;63:486-500.

12. Edwards RF, Harrison TM, Davis SM. Potentially inappropriate prescribing for geriatric inpatients: an acute care of the elderly unit compared to a general medicine service. Consult Pharm. 2003;18(37-42):47-9.

13. Lee JK, Slack MK, Martin J, Ehrman C, Chisholm-Burns M. Geriatric patient care by US pharmacists in healthcare teams: systematic review and meta-analyses. J Am Geriatr Soc. 2013;61:1119-27.

14. Walsh KA, O'Riordan D, Kearney PM, Timmons S, Byrne S. Improving the appropriateness of prescribing in older patients: a systematic review and meta-analysis of pharmacists' interventions in secondary care. Age Ageing. 2016;45:201-9.

15. Limpawattana P, Kamolchai N, Theeranut A, Pimporn J. Potentially inappropriate prescribing of Thai older adults in an internal medicine outpatient clinic of a tertiary care hospital. Afr J Pharm Pharmacol. 2013;7:2417-22.

16. Al-Azayzih A, Alamoori R, Altawalbeh SM. Potentially inappropriate medications prescribing according to Beers criteria among elderly outpatients in Jordan: a cross sectional study. Pharm Pract (Granada). 2019;17(2):1439. https://doi.org/10.18549/PharmPract .2019.2.1439.

17. Awad A, Hanna O. Potentially inappropriate medication use among geriatric patients in primary care setting: A cross-sectional study using the Beers, STOPP, FORTA and MAI criteria. PLoS ONE. 2019;14:e0218174. https://doi.org/10.1371/journ al.pone. 0218174 .

18. Blanc AL, Spasojevic S, Leszek A, Théodoloz M, Bonnabry P, Fumeaux T, Schaad N. A comparison of two tools to screen potentially inappropriate medication in internal medicine patients. $\mathrm{J}$ Clin Pharm Ther. 2018;43:232-9.

19. Egger SS, Bachmann A, Hubmann N, Schlienger RG, Krähenbühl S. Prevalence of potentially inappropriate medication use in elderly patients comparison between general medical and geriatric wards. Drugs Aging. 2006;23:823-37.

20. Shaw PB, Delate T, Lyman A Jr, Adams J, Kreutz H, Sanchez JK, Dowd MB, Gozansky W. Impact of a clinical pharmacy specialist in an emergency department for seniors. Ann Emerg Med. 2016;67:177-88.

21. van der Stelt CAK, Windsant-van den Tweel AMAV, Egberts ACG, van den Bemt PMLA, Leendertse AJ, Hermens WAJJ, van Marum RJ, Derijks HJ. The association between potentially inappropriate prescribing and medication-related hospital admissions in older patients: a nested case control study. Drug Saf. 2016;39:79-87.

22. Prasert V, Akazawa M, Shono A, Chanjaruporn F, Ploylearmsang C, Muangyim K, Wattanapongsatit T, Sutin U. Applying the Lists of Risk Drugs for Thai Elderly (LRDTE) as a mechanism to account for patient age and medicine severity in assessing potentially inappropriate medication use. Res Soc Adm Pharm. 2018;14:451-8.

23. Fu AZ, Jiang JZ, Reeves JH, Fincham JE, Liu GG, Perri M III. Potentially inappropriate medication use and healthcare expenditures in the US community-dwelling elderly. Med Care. 2007;45:472-6.

24. McMahon CG, Cahir CA, Kenny RA, Bennett K. Inappropriate prescribing in older fallers presenting to an Irish emergency department. Age Ageing. 2014;43:44-50.

25. Cool C, Cestac P, Laborde C, Lebaudy C, Rouch L, Lepage B, Vellas B, de Souto BP, Rolland Y, Lapeyre-Mestre M. Potentially inappropriate drug prescribing and associated factors in nursing homes. J Am Med Dir Assoc. 2014;15:850e1-e9. https://doi. org/10.1016/j.jamda.2014.08.003.

26. Pannoi T, Chapman RS, Panza A. Prevalence of potentially inappropriate medication (PIM) and factors associated with PIM in elderly outpatient prescriptions at a district hospital in the southern region of Thailand. J Health Res. 2014;28:100-8.

27. Gallagher P, O'Mahony D. STOPP (Screening Tool of Older Persons' potentially inappropriate Prescriptions): application to acutely ill elderly patients and comparison with Beers' criteria. Age Ageing. 2008;37:673-9.

28. Wilt TJ, MacDonald R. Doxazosin in the treatment of benign prostatic hypertrophy: an update. Clin Interv Aging. 2006;1:389-401.

29. Chapple CR. Selective alpha 1-adrenoceptor antagonists in benign prostatic hyperplasia: rationale and clinical experience. Eur Urol. 1996;29:129-44.

30. Lepor H. Alpha blockers for the treatment of benign prostatic hyperplasia. Rev Urol. 2007;9:181-90.

31. Krumholz A, Wiebe S, Gronseth GS, Gloss DS, Sanchez AM, Kabir AA, Liferidge AT, Martello JP, Kanner AM, Shinnar S, Hopp JL, French JA. Evidence-based guideline: management of an unprovoked first seizure in adults: report of the guideline development subcommittee of the American Academy of Neurology and the American Epilepsy Society. Epilepsy Curr. 2015;15:144-52.

32. Osborne A, Taylor L, Reuber M, Grünewald RA, Parkinson M, Dickson JM. Pre-hospital care after a seizure: evidence base and United Kingdom management guidelines. Seizure. 2015;24:82-7.

33. Kirby M, Denihan A, Bruce I, Radic A, Coakley D, Lawlor BA. Benzodiazepine use among the elderly in the community. Int $\mathbf{J}$ Geriatr Psychiatry. 1999;14:280-4.

34. Wang PS, Bohn RL, Glynn RJ, Mogun H, Avorn J. Hazardous benzodiazepine regimens in the elderly: effects of half-life, dosage, and duration on risk of hip fracture. Am J Psychiatry. 2001;158:892-8.

35. Pasina L, Djade CD, Tettamanti M, Franchi C, Salerno F, Corrao S, Marengoni A, Marcucci M, Mannucci PM, Nobili A, REPOSI Investigators. Prevalence of potentially inappropriate medications and risk of adverse clinical outcome in a cohort of hospitalized elderly patients: results from the REPOSI Study. J Clin Pharm Ther. 2014;39:511-5.

36. Lin H-Y, Liao C-C, Cheng S-H, Wang P-C, Hsueh Y-S. Association of potentially inappropriate medication use with adverse outcomes in ambulatory elderly patients with chronic diseases. Drugs Aging. 2008;25:49-59.

37. Dalleur O, Boland B, Losseau C, Henrard S, Wouters D, Speybroeck N, Degryse JM, Spinewine A. Reduction of potentially inappropriate medications using the STOPP criteria in frail older inpatients: a randomised controlled study. Drugs Aging. 2014;31:291-8.

38. Gallagher PF, O'Connor MN, O’Mahony D. Prevention of potentially inappropriate prescribing for elderly patients: a randomized controlled trial using STOPP/START criteria. Clin Pharmacol Ther. 2011;89:845-54.

39. Hill-Taylor B, Walsh KA, Stewart S, Hayden J, Byrne S, Sketris IS. Effectiveness of the STOPP/START (Screening Tool of Older Persons' potentially inappropriate Prescriptions/Screening Tool to Alert doctors to the Right Treatment) criteria: systematic review and meta-analysis of randomized controlled studies. J Clin Pharm Ther. 2016;41:158-69. 